\title{
Advantages of pulse-shaping applied to OFDM systems over underwater acoustic channels
}

\author{
Said Lmai, Arnaud Bourré, Christophe Laot and Sébastien Houcke \\ Institut Mines-Telecom ; Telecom Bretagne ; UMR CNRS 6285 Lab-STICC \\ Université Européenne de Bretagne, \\ Technople Brest-Iroise - CS 83818 - 29238 Brest Cedex 3, France \\ E-mail: said.lmai@telecom-bretagne.eu
}

\begin{abstract}
Orthogonal frequency division multiplexing (OFDM) systems combat inter-symbol interferences (ISI) using cyclic prefix (CP) or the equivalent guard interval (GI) in case of zero-padded (ZP) OFDM. Nevertheless, inter-carrier interferences (ICI) due to the synchronization mismatches for instance, remain a strong disadvantage. Pulse-shaping is one of the solutions introduced in narrow band context. In this paper, we explored the use of this technique in wideband environments such underwater acoustic (UWA) channel. For this purpose, we developed an appropriate pulse-shaped CP-OFDM scheme. In addition to ICI reduction over channels with Doppler shift and spread, the proposed system, compared to the conventional CP-OFDM, achieves at equal transmit power important $E_{b} / N_{o}$ savings: the longer the $\mathrm{CP}$, the greater the saving. Unlike existing pulse-shaped OFDM designs, we propose to share out pulse-shaping between transmission and reception modules. Results from simulations are corroborated by sea trials.
\end{abstract}

\section{INTRODUCTION}

Transmission over underwater acoustic (UWA) channels is challenging, since it faces large time-delay spread, wide Doppler spread and limited bandwidth having the same order of magnitude as that of carrier frequencies. UWA communication is wideband in nature and Doppler effects are frequency dependant, unlike narrowband systems which experience an approximately constant carrier frequency offset. In recent years, UWA communications have taken advantage of special focus on orthogonal frequency division multiplexing (OFDM) schemes [1]-[3]. Known for its simple implementation in general, OFDM system combats inter-symbol interferences (ISI). The symbol duration therein is larger than the multipath delay of the channel. The most common OFDM design is cyclic prefix (CP) OFDM. CP is last part copy of the OFDM symbol that is pre-pended to the transmitted symbol. The CP length is greater than the channel delay spread. At the receiver, $\mathrm{CP}$ is merely removed in order to run the fast convolution algorithm based on the FFT method [4], [5]. This enables conducting a simple equalization. Regarding zero-padded (ZP) OFDM, each OFDM symbol is rather post-pended with zeros which constitute the guard interval (GI). For both versions ( $\mathrm{CP}$ and $\mathrm{ZP}$ ), it is customary to insert null subcarriers in the OFDM symbol according to predefined patterns. Disposed between pilot and data subcarriers [2], [3], they are designed for different uses: e.g. estimation of mean Doppler shift.

Conventional OFDM systems suffer from certain drawbacks. CP length for CP-OFDM or corresponding GI length for ZP-OFDM, is often over-dimensioned since channel statistics estimation is not accurate, especially over time-varying channels such UWA channel. It should be noted that CP use decreases spectral efficiency in proportion to its size. Therefore, while maintaining a given Bit Error Rate (BER) value, increasing the size of $\mathrm{CP}$ leads to an augmentation in the average signal-to-noise ratio (SNR) per bit $\left(E_{b} / N_{o}\right)$. Furthermore, OFDM modulation is sensitive to inter-carrier interferences (ICI) that arise at the receiver, mainly because of transmitter/receiver mobility and channel variability. Classic schemes are not robust to Doppler effects, particularly in wideband systems. Among the techniques used to reduce ICI, [6] studied the pulse-shaping in narrow band context and over AWGN channels. [2] introduced its use in wideband system with Doppler spread. The pulses commonly used are Nyquist windows where zeros are inserted on both sides of the useful OFDM signal before performing the pulse-shaping (also named apodization). However, the pulse-shaping may be implemented in different ways: one can favor for instance to use CP-OFDM or ZP-OFDM and to focus apodization on the transmitter module and/or at the receiver part.

In this paper, we propose a particular CP-OFDM pulseshaping design and investigate the performance against models already discussed in the literature. In addition to ICI reduction over channels with Doppler shift and spread, other metrics of interest are considered for comparison (e.g. $E_{b} / N_{o}$ savings). The suggested system performs a balanced repartition of the pulse-shaping in time domain, between transmission and reception modules. Information contained in $\mathrm{CP}$ is carefully used when exploiting the entire signal observed at the receiver. Unlike aforementioned pulse-shaping schemes, no zeros are inserted on both sides of the useful OFDM signal before operating apodization. Thus, it achieves more interesting spectral efficiency and consequently higher throughput.

After considering the interest of the introduced pulseshaping on reducing ICI in narrowband systems, our scheme is applied in wideband context. In such environment, time variations in the channel and transmitter/receiver movement engender significant frequency-dependent Doppler spread and shift. Close-by a perfect time synchronization at the begining of the OFDM symbol, we have identified the gains of applying the proposed OFDM design in terms of BER performance. Moreover, increasing the size of CP does not occasion any 
loss in SNR per bit.

In order to explore approaches which handle pulse-shaping in OFDM schemes at the transmitter [2], [7] or at the receiver part only [8], we carried out the implementation of our scheme restricted to one side (transmission or reception). We find that pulse-shaped OFDM with equi-distributed apodization between transmitter and receiver shows best BER performance. Likewise, IFFT and FFT operations are employed and no meaningful complexity is added.

The remainder of the paper is organized as follows. Section II is devoted to describing the system model and tracing the main motivations. We introduce several simulation scenarios and give results in Section III. Section IV presents experimental results. Finally, conclusions (and future work) are summarized in Section V.

\section{SYSTEM MODEL}

We build up our OFDM system based on the pulse-shaping function $\sqrt{g(t)}$ implemented at both sides: transmission and reception modules. Conventional CP-OFDM system with rectangular window is denoted Rect-OFDM, whereas RRCOFDM is used to refer to the pulse-shaped OFDM system with the root raised cosine window $\sqrt{g(t)}$. The time-continuous passband $\mathrm{N}$-subcarriers symbol of duration $T=T_{o}+T_{g}$, is expressed as :

$$
s(t)=\sum_{k=0}^{N-1} d_{k} \sqrt{g(t)} e^{j 2 \pi f_{k} t}, t \in\left[0, T_{o}+T_{g}\right]
$$

where :

- $T_{o}($ resp. $N)$ and $T_{g}\left(\right.$ resp. $\left.N_{c p}\right)$ denote the original useful signal duration (resp. number of samples) and the CP length (resp. number of samples) respectively (for a sampling interval $\left.T_{s}=T_{o} / N\right)$

- $d_{k}$ is the information symbol (of duration $T_{s}$ ) transmitted on the $k^{t h}$ subcarrier (whose frequency is $f_{k}$ ), chosen from a predefined constellation diagram such as phaseshift keying (PSK) or quadrature amplitude modulation (QAM)

- $f_{k}-f_{m}=\frac{k-m}{T_{o}}$ and $\frac{1}{T_{o}}$ is the subcarrier spacing

- $B=\frac{N}{T_{o}}$ is the nominal bandwidth

- $\sqrt{g(t)}$ is the root raised cosine and $g(t)$ is defined by

$$
g(t)= \begin{cases}1, & |t|<\frac{T_{o}(1-\alpha)}{2} \\ \frac{1}{2}\left\{1+\cos \left(\frac{\pi}{\alpha T_{o}}\left(|t|-\frac{T_{o}(1-\alpha)}{2}\right)\right)\right\}, & \frac{T_{o}(1-\alpha)}{2} \leq|t|<\frac{T_{o}(1+\alpha)}{2} \\ 0, & \text { otherwise }\end{cases}
$$

$g(t)$ is a finite duration Nyquist pulse unlike the usual Nyquist filters with compact support in frequency domain. The length of $\sqrt{g(t)}$ is accurately the length of one OFDM symbol. Regarding this point, apodization operation is performed just over the whole OFDM symbol, CP included, in such way the roll-off period length corresponds exactly to $T_{g}$ i.e. $T_{g}=\alpha T_{o}$,

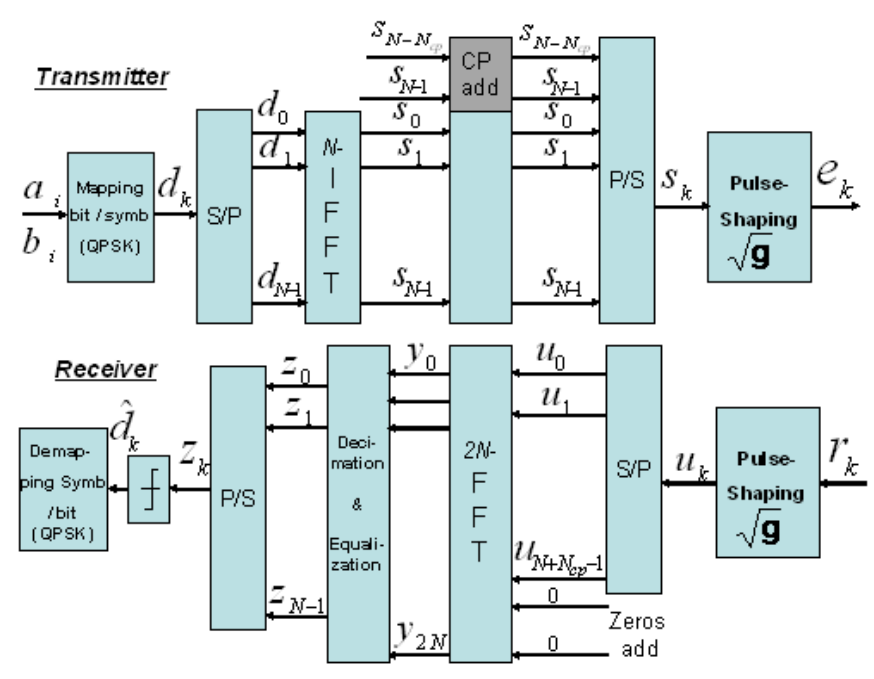

Fig. 1. Pulse-shaped OFDM scheme

where $\alpha$ is the roll-off factor of the root pulse function $\sqrt{g(t)}$ used for apodization.

A simple implementation scheme of the OFDM modulation consists in mapping data bits $a_{i}, b_{i}$ into information symbols $d_{k}$. Afterwards, IFFT is performed on these symbols after converting them from single stream to $N$ parallel ones. The $\mathrm{CP}$, which is last part copy of the original useful OFDM symbol is added.

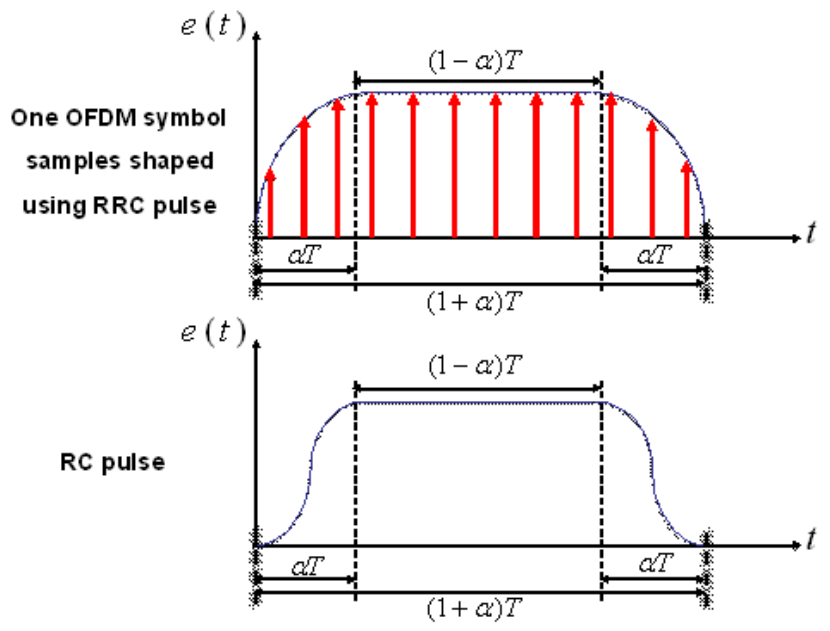

Fig. 2. Root Raised Cosine and Raised Cosine time domain pulses illstration for one CP-OFDM symbol

As regards our scheme, one can notice that the OFDM symbol samples obtained in time domain (Fig.2), are of low intensity at the edges of the symbol, unlike those on the base plateau which carry more energy. Over an ideal AWGN channel and in the absence of frequency offset, the received signal is :

$$
r(t)=\sum_{k=0}^{N-1} d_{k} \sqrt{g(t)} e^{j 2 \pi f_{k} t}+n(t), t \in\left[0,(1+\alpha) T_{o}\right]
$$


where $n(t)$ is the ambient Gaussian noise. Minimum-error correlation demodulator is employed. For the transmitted symbol $d_{n}$ on the $n^{\text {th }}$ subcarrier, the demodulator decides :

$$
\hat{d}_{n}=\int_{-\infty}^{+\infty} r(t) \sqrt{g(t)} e^{-j 2 \pi f_{n} t} d t
$$

For simple practical implementation at the receiver module, first the symbol is shaped using the same pulse $\sqrt{g(t)}$. Next, $N-N_{c p}$ zeros are padded at the end of the received OFDM symbol before applying $2 N$-FFT. Since the original transmitted data are carried on sub-channels $f_{k}\left(\frac{1}{T_{o}}\right.$ spaced $)$, the output of the FFT operation is down sampled at rate 1/2. In case of perfect channel estimate, one-tap simple equalization is then achieved, otherwise elaborates structures might be used [9].

A simple architecture of the system is described in Fig.1. In the literature, in addition to the guard interval, pulse-shaping introduces further interval [2], [7]. This interval is nothing else than the roll-off time of the used pulse function. It is made up after extending the original useful OFDM symbol (of duration $T_{o}$ ) by inserting zeros at both sides.

With respect to Rect-OFDM, the transmitted timecontinuous OFDM symbol is given by the expression :

$$
e(t)=\sum_{k=0}^{N-1} d_{k} \operatorname{rect}(t) e^{j 2 \pi f_{k} t}, t \in\left[0, T_{o}+T_{g}\right]
$$

where : $\operatorname{rect}(t)$ is a rectangular pulse in time with unit amplitude and duration $T=T_{o}+T_{g}$. Thereby, at the receiver side, reverse operations begin by removing the $\mathrm{CP}$ and operate FFT on the $N$ parallel streams. Afterwards, the receiver carries out one-tap simple equalization before proceeding to data detection and decision.

Our initial motivations to take on pulse-shaping are:

- on the transmitted CP-OFDM symbol, although CP is a redundancy in time of a part of original information, towards the noise, it will be affected differently. This somehow offers a certain diversity. So, it would be useful to exploit, at the reception side, data contained in the $\mathrm{CP}$

- for a given average SNR, pulse-shaping reduces the energy, in time domain, on the edges of the OFDM symbol designed and allows to place the ISI in signal regions of low SNR

- the implementation of pulse-shaping at the receiver side mitigates even more the energy of ISI-affected samples. Indeed, weighting coefficients of the apodization function are lower at the edges

- provided that the average ICI power depends on the spectral magnitudes of $g(t)$ [6], frequency spectrum of the used apodization function has smaller side-lobes than sinc (which corresponds to the rectangular pulse)

- by allowing a gradual and smooth increase of signal amplitude, pulse-shaping avoids sudden changes occurred when using rectangular pulse

\section{SimUlation RESUltS}

In order to better visualize the effects of frequency offset and Doppler scale, we conduct baseband simulations using $N=64$. Due to high Doppler spread in UWA channel, the packet size typically used is short. Large channel impulse response (CIR) leads to consider a $\mathrm{CP}$ length intended to operate under different environment conditions. We put on at least two values for CP length. Besides, we consider the QPSK modulation scheme. We generate randomly one OFDM symbol and one corresponding channel and additive white gaussian noise (AWGN) realization. Monte Carlo simulations are run to estimate the BER value for each of the $2 \times 10^{4}$ OFDM symbol realizations. The multipath channel model is selected to be a Rayleigh fading model with an exponentially decaying power profile. It consists of 10 discrete paths $T_{s}$ spaced with decaying normally distributed random amplitudes. Performance comparaison between the two systems is made at equal transmitted energies.

First, we sought to assess CP length influence on $E_{b} / N_{o}$. In addition to $N=64, N=128$ is used for more clarification. Next, we perform simulations in narrow band context, where frequency offset is the same on all subcariers. Some wideband synchronization techniques start by converting the issue to narrow band before correcting the resulting frequency offset [10]. We intentionally introduce several values of frequency offsets and measure the corresponding BER for both: Rect-OFDM and RRC-OFDM systems. Afterwards, the wideband context is considered to compare the two schemes strengths. The Doppler effect is mainly due to the transmitter/receiver motion. We explore the BER degradations according to different Doppler scales over one OFDM block. At the begining of each OFDM symbol, perfect time sychronization and a one-tap post-FFT equalizer with perfect channel estimates are assumed. Note that for both schemes, no channel coding is used.

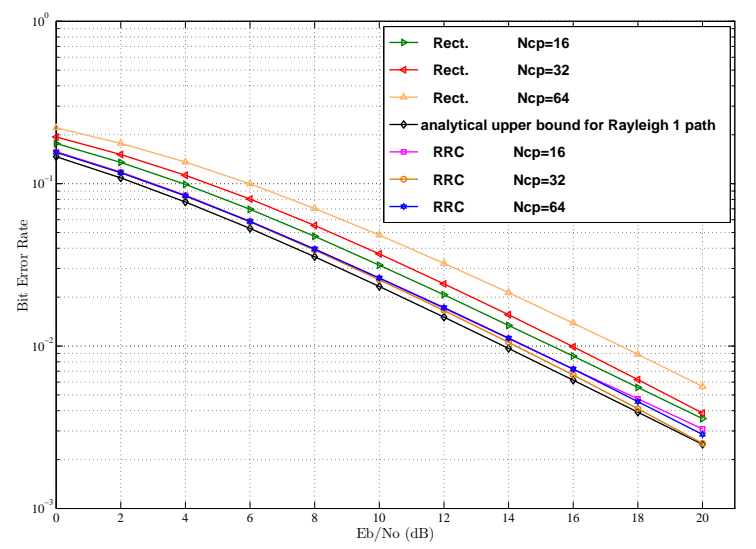

Fig. 3. BER performance of RRC-OFDM vs Rect-OFDM over rayleigh channel $(\mathrm{N}=64)$ 


\section{A. CP length influence on $E_{b} / N_{o}$}

UWA communications face inherent time-varying channel with relatively large CIR. In order to combat ISI, CP length should be at least, as long as the channel delay spread. Owing to the fact that channel statistics estimation is not usually accurate, the used CP lengths in OFDM systems are set to large values. However, channel throughput relies on the bandwidth efficiency which can be expressed as: $\frac{N}{N+N_{c p}}$. Therefore, for $\mathrm{M}$-ary modulation, the relationship between the SNR per bit $\left(E_{b} / N_{o}\right)$ and the SNR per symbol $\left(E_{s} / N_{o}\right)$ is determined by:

$$
E_{b} / N_{o}=E_{s} / N_{o}-10 \log _{10}\left(\log _{2}(M) \cdot \frac{N}{N+N_{c p}}\right)
$$

where $E_{s}$ is the energy per symbol and $\mathrm{M}$ is the modulation order.

$N=64$ might give rise, even with large CPs, to penalizing ISI effect especially in case of large CIR. Indeed, significant portion of 64-subcarriers symbol is corrupt. To address this problem, we headed towards $N=128$ (and more over sea trials in section IV). Performance from comparison between Rect-OFDM and RRC-OFDM uncoded systems are plotted in terms of BER taken as a function of $E_{b} / N_{o}$. Resulting curves are shown in Figures 3 and 4. Simulation parameters are $N_{c p}=16,32$ and 64 for $N=64$ and $N_{c p}=32,64$ and 128 for $N=128$. The analytical upper bound for rayleigh one path channel is plotted as reference.

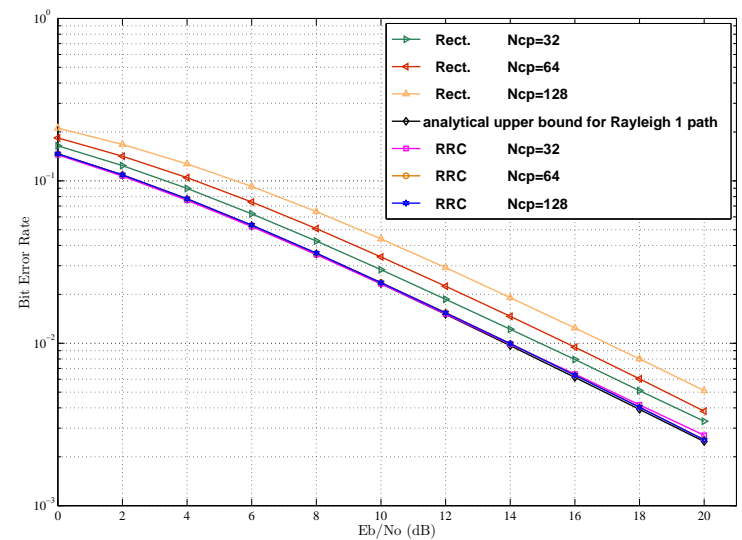

Fig. 4. BER performance of RRC-OFDM vs Rect-OFDM over rayleigh channel $(\mathrm{N}=128)$

In both Figures 3 and 4, the first thing to note is that as the Rect-OFDM CP length increases performance degrades. In particular, with respect to $N=64$ one can observe for $B E R=10^{-2}$ that $E_{b} / N_{o}$ in case of $N_{c p}=32$ is more than $1 d B$ lower than that corresponding to $N_{c p}=64$. Nevertheless, the gap is rather more than $2 d B$ when considering $N_{c p}=64$ and $N_{c p}=128$ for $N=128$. This means that the use of large CP to take up long CIR leads to an "important" loss in $E_{b} / N_{o}$. In contrast to these results, when $N=64$ the RRCOFDM scheme achieves for $B E R=10^{-2}$ and $N_{c p}=16$ an
$E_{b} / N_{o}$ nearly $1 d B$ lower than that of Rect-OFDM with the same CP length. The gain is about $2 d B$ in case of $N=128$ and $N_{c p}=64$.

Moreover, if the particular ISI effect is taken into account when $N=64$, increasing CP length to $N_{c p}=32$ and $N_{c p}=$ 64 for the first case $(N=64)$ and to $N_{c p}=64$ and $N_{c p}=$ 128 for the second one $(N=128)$ does not occasion almost any additional loss in $E_{b} / N_{o}$. One can deduce that the RRCOFDM system provides valuable savings in terms of $E_{b} / N_{o}$.

Furthermore, unlike the Rect-OFDM scheme, RRC-OFDM reaches almost the analytical upper bound for rayleigh one path channel. This is due to the fact that the entire energy carried by the OFDM symbol (CP included) is exploited. The RRC-OFDM is a quite optimized scheme in such time-varying channels.

\section{B. Narrow band context}

In this context, the average ICI power for a certain information symbol $d_{k}$ depends on two parameters [6]: the number of the subcarriers $N$ and the spectral magnitudes of the pulse-shaping function $g(t)$ at the frequencies $\left(\left((l-k) / T_{o}\right)+\Delta f\right), l \neq k, l=0,1, \ldots, N-1$. We handle simulations for normalized frequency offset values ranging from $\Delta f . T_{o}=0.05$ to $\Delta f . T_{o}=0.15$. Results for average SNR per bit $\left(E_{b} / N_{o}\right)$ of 16 and $20 d B$ are given. $N=64$ and the two CP length values considered are: $N_{c p}=16$ and $N_{c p}=32$. For each case the BER is taken as a function of the frequency offset. Resulting curves are presented in Figures 5 and 6. Comparing the performance of the two schemes, we observe that the performance of the RRC-OFDM is better in all cases. In particular, for $E_{b} / N_{o}=16 d B, N_{c p}=16$ and $\Delta f . T_{o}$

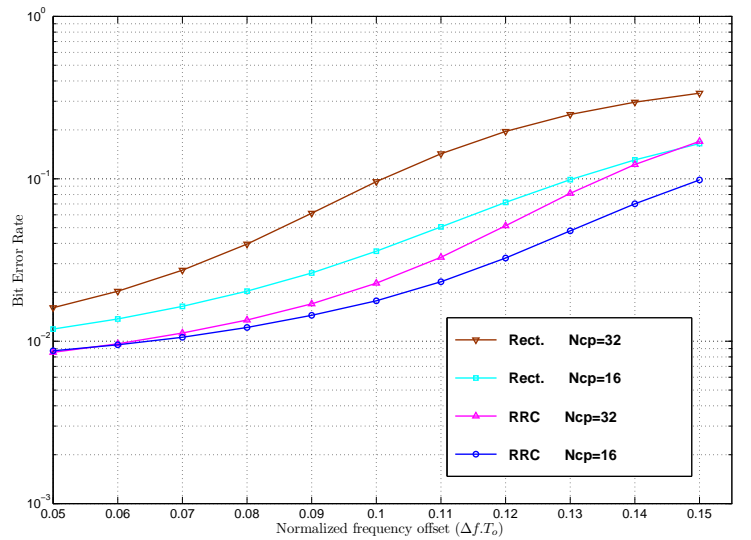

Fig. 5. BER performance in the case of $E_{b} / N_{o}=16 d B$, uniform frequency offset

around 0.12, RRC-OFDM achieves a BER at least two times smaller than that of the Rect-OFDM. Moreover, for $N_{c p}=32$, the RRC-OFDM BER performance is more than three times smaller, as seen in Fig.5. $E_{b} / N_{o}=20 d B$ leads to identify a larger gap between the two systems. Fig.6 shows that BER value is widely five times smaller for $\Delta f . T_{o}$ around 0.12 and $N_{c p}=32$ while using RRC-OFDM. 


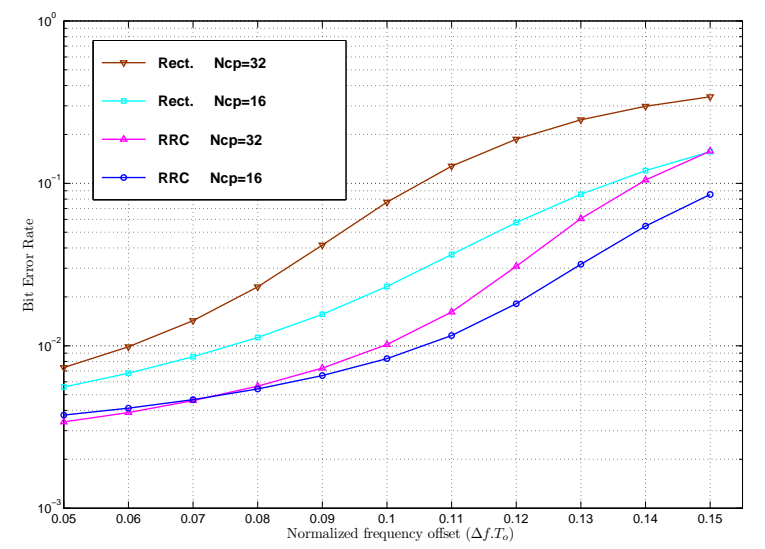

Fig. 6. BER performance in the case of $E_{b} / N_{o}=20 \mathrm{~dB}$, uniform frequency offset

If we take the two curves of RRC-OFDM in Fig.6, we can see that they intersect at a value of $\Delta f . T_{o}$ around 0.07 . This is because below this value, the power of the main lobe at the concerned subcarrier outweighs the contribution of the two parameters involved in narrow band ICI context, namely: the power of the other subcarriers side lobes and the value of the frequency offset. Besides, on both ends of the normalized frequency offset interval considered, we can notice that the gain is low. This let us conclude that for relatively small and high frequency offset values, RRC-OFDM simply achieves $E_{b} / N_{o}$ saving.

\section{Wideband context}

In wideband environment, Doppler effect is induced by transmitter/receiver movement combined to channel variability. Thus, the transmitted signal experiences severe distortions which might be depicted by the ratio (referenced Doppler scale or scaling factor): $\frac{v}{c}$, where $v$ is the transmitter/receiver relative speed and $c$ is the sound velocity. When compared to narrow band case, this frequency dependent phenomenon is more complex to cope with. In our simulations, Doppler scale is assumed to be constant over the duration of one OFDM symbol. Again, the two CP lengths employed are: $N_{c p}=16$ and $N_{c p}=32$. BER performance is taken as a function of Doppler scaling factor. This factor is described by values taken in the range from $2.10^{-3}$ to $8.10^{-3}$ which corresponds, in the tests conducted, to motion at maximum relative speed of $12 \mathrm{~m} / \mathrm{s}$. Results for $E_{b} / N_{o}$ of 16 and $20 \mathrm{~dB}$ are depicted in Figures 7 and 8 .

In the case of $E_{b} / N_{o}=16 d B$ and for a Doppler scale of $5.10^{-3}$, Fig. 7 shows that RRC-OFDM achieves a BER slightly more than three times smaller than that of the Rect-OFDM for $N_{c p}=32$. Furthermore, we can easily identify in Fig. 8 that the gain is larger. In particular, RRC-OFDM reaches a BER more than four times smaller than that of the Rect-OFDM for a Doppler scale of $5.10^{-3}$ and $N_{c p}=32$. It is obvious that the RRC-OFDM system outperforms in all cases the Rect-OFDM one.

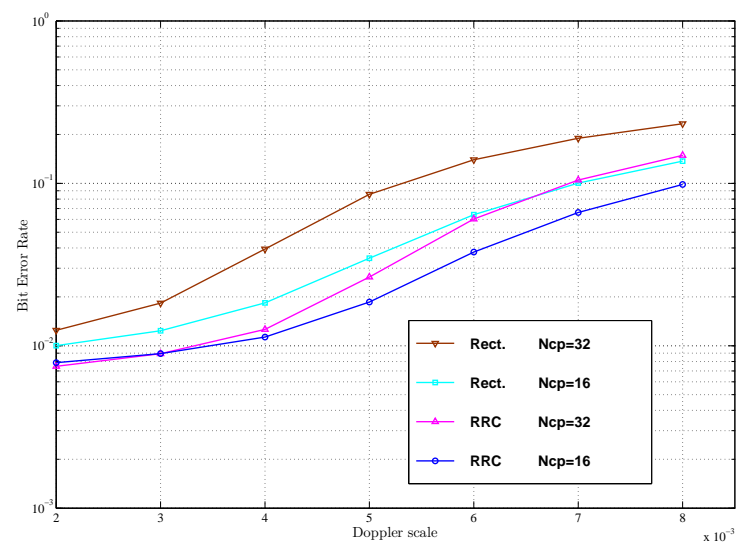

Fig. 7. BER performance in the case of $E_{b} / N_{o}=16 \mathrm{~dB}$, frequencydependant Doppler scale

Studies in literature have introduced several techniques [10], [11] for synchronization in the presence of Doppler spread and shift. Scenarios treated therein concern maximum relative speeds around $5 \mathrm{~m} / \mathrm{s}$ with obversed Doppler scaling factors well bellow $6.10^{-3}$. In Fig.8, the range of relatively low Doppler scales (bellow $6.10^{-3}$ ) allows to observe the robustness of the RRC-OFDM scheme in wideband environments. Nevertheless, performance for high Doppler scaling factors,

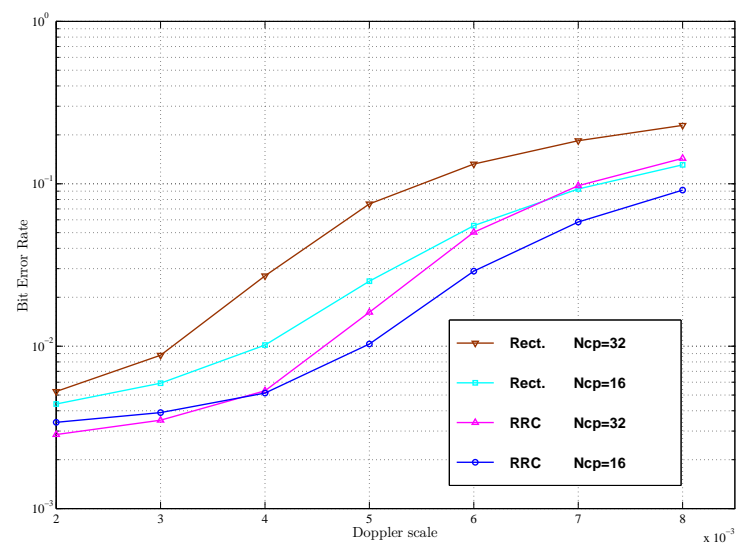

Fig. 8. BER performance in the case of $E_{b} / N_{o}=20 \mathrm{~dB}$, frequencydependant Doppler scale

particularly for $7.10^{-3}$ or more, let infer that RRC-OFDM simply achieves $E_{b} / N_{o}$ saving in this zone. The same is valid for very small values (below3.10 ${ }^{-3}$ ).

\section{Pulse-shaping applied to a single side}

After implementing equi-distributed apodization between transmitter and receiver in previous subsections, we investigate the use of pulse-shaping at the transmitter (or receiver) module only. Otherwise, instead of performing the repartition of the pulse function between the transmission and reception sides, it is used exclusively at the transmitter (or receiver) part. Let 
$\mathrm{RC} / \mathrm{Tx}(\mathrm{Rx})$ designates the new scheme. Fig.9 shows BER performance achieved compared to balanced apodization scheme result for $N_{c p}=32,64$ and 128 in case of $N=128$. One can observe that RRC-OFDM acheives slightly less than $1 d B$ BER improvment for $N_{c p}=32$. Furthermore, additionnal gain is identified when increasing the CP length as regards to $E_{b} / N_{o}$ savings. Therefore, while RRC-OFDM system preserves same

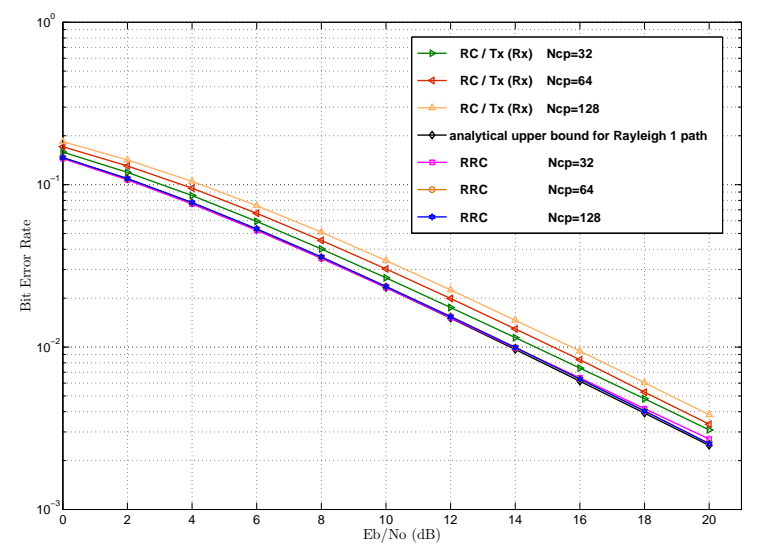

Fig. 9. BER performance of RRC-OFDM vs Rect-OFDM over rayleigh channel $(\mathrm{N}=128)$ while pulse-shaping restricted to the transmitter side

performance for $N_{c p}=64$ and $128, \mathrm{RC} / \mathrm{Tx}(\mathrm{Rx})$ scheme results degrade gradually as $\mathrm{CP}$ length increases.

\section{EXPERIMENTAL RESULTS}

In order to test our scheme, we conducted an experiment in shallow water of Brest harbor, France on June $13^{d}, 2012$. Experiment parameters are as follows: Differential Quadrature

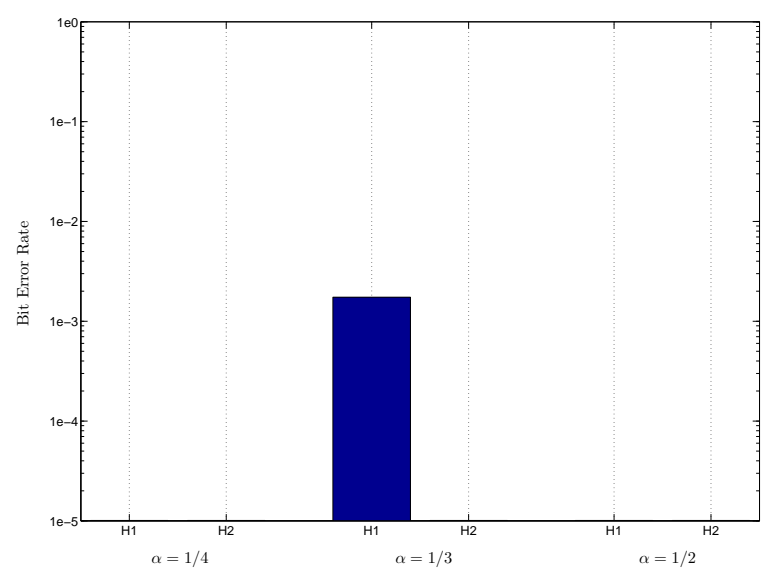

Fig. 10. RRC-OFDM performance for $N=60$, where $1 e-5$ represents zero error

Phase Shift Keying (D-QPSK) modulation, rate 1/2 punctured convolutional coding and three numbers of subcarriers 60 , 120 and 240 over a bandwidth of $4 \mathrm{kHz}$. D-QPSK modulation is adopted as there is no need for phase reference and low complexity blind channel estimation method is used. The distance between the transmitter (ITC1001) and the receiver is about $900 \mathrm{~m}$ and two omni-directional hydrophones ( $B \& K 8106)$ were used for receiving (without multiple inputs processing). The first and the second hydrophones are designated by $H 1$ and $H 2$ respectively. The transmit power is $P=170 d B / / 1 \mu P a$ at 1 meter and the carrier frequency is $f_{c}=18 \mathrm{kHz}$. Transmission is carried out using one tranducer and the hydrophones were separated by about $10 \lambda$. Regarding the GI of duration $T_{g}$, three values of $\alpha$ where $T_{g}=\alpha T_{o}$ were tested: $\alpha=1 / 4,1 / 3$ and $1 / 2$. Sea trials transmissions concern superframes sizing 48818 bits. Results synthesis is summarized in Figures 10 and 11. It should be noted that there

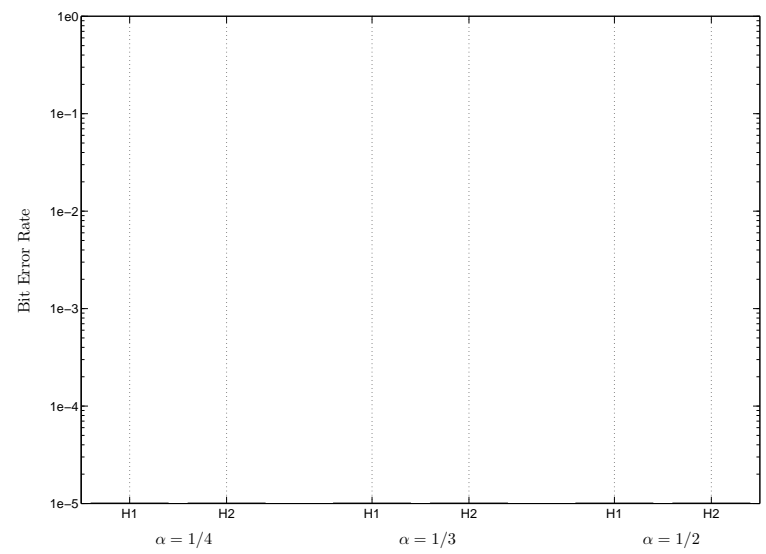

Fig. 11. RRC-OFDM performance for $N=120$ and 240 , where $1 e-5$ represents zero error

was intense activity in the harbor which introduce considerable variations in the channel and consequently the destruction of the subcarriers orthogonality. $N=60$ and $\alpha=1 / 3$ is the unique case where few errors occur. Since for shorter GI ( $\alpha=1 / 4$ ) the system operates with zero erro, it's may be due to a very close and intensive noise resounding. Figures 10 and 11 show that the entire data are received without errors in all other configurations. One can deduce that, despite existing environmental noise sources, the RRC-OFDM system is working properly.

\section{CONCLUSIONS}

A pulse-shaped OFDM structure has been simulated and tested over a real UWA time-varying channel. Through simulations we pinpoint for different sizes of CP, precious $E_{b} / N_{o}$ savings achieved by RRC-OFDM system compared to the Rect-OFDM. Then, we identify the advantages of OFDM pulse-shaping in narraow band and wideband contexts. Balanced apodization between transmission and reception modules shows interesting BER performance. Sea trials have confirmed the robustness of the proposed scheme.

Such promising performance will push us to think about expanding the size of transmitted data and introduce the use of the proposed scheme in formal configurations (e.g. a network scenario). 


\section{REFERENCES}

[1] S. Mason, C. Berger, S. Zhou, K. Ball, L. Freitag, and P. Willett, "An ofdm design for underwater acoustic channels with doppler spread", in Proc. 13th IEEE Digit. Signal Process. Workshop / 5th IEEE Signal Process. Edu. Workshop, Marco Island, FL, pp. 138 -143, Jan. 2009.

[2] Z. Wang, S. Zhou, G.B. Giannakis, C.R. Berger, and J. Huang, "Frequency-domain oversampling for zero-padded ofdm in underwater acoustic communications", IEEE J. Oceanic Eng., vol. 37, no. 1, pp. $14-24$, Jan. 2012

[3] C.R. Berger, J. Gomes, and J.M.F. Moura, "Study of pilot designs for cyclic-prefix ofdm on time-varying and sparse underwater acoustic channels", in Proc. MTS/IEEE OCEANS Conf., Santander, Spain, June 2011.

[4] B. Muquet, Z. Wang, G.B. Giannakis, M. de Courville, and P. Duhamel, "Cyclic prefixing or zero padding for wireless multicarrier transmissions?", IEEE Trans. Commun., vol. 50, no. 12, pp. 2136 - 2148, Dec. 2002.

[5] M. Borgerding, "Turning overlap-save into a multiband mixing, downsampling filter bank", IEEE Signal Process. Mag., vol. 23, no. 2, pp. $158-161$, Mar. 2006.

[6] P. Tan and N.C. Beaulieu, "Communication theory analysis of the effects of nyquist pulse-shaping on the performance of ofdm systems with carrier frequency offset", Eur. Trans. Telecommun., vol. 20, no. 1, pp. 9-22, Jan. 2009

[7] N.C. Beaulieu and P. Tan, "Effect of transmitter nyquist shaping on ici reduction in ofdm systems with carrier frequency offset", IEE Electron. Lett., vol. 41, no. 13, pp. 746 - 748, June 2005.

[8] N.C. Beaulieu and Peng Tan, "On the effects of receiver windowing on ofdm performance in the presence of carrier frequency offset", IEEE Trans. Wireless Commun., vol. 6, no. 1, pp. 202 -209, Jan. 2007.

[9] B. Geller, J.M. Broissier, and V. Capellano, "Equalizer for high data rate transmission in underwater communications", in Proc. Oceanic Eng. \& SEE IEEE OCEANS Conf., Brest, France, sep. 1994.

[10] Baosheng Li, Shengli Zhou, M. Stojanovic, L. Freitag, and P. Willett, "Multicarrier communication over underwater acoustic channels with nonuniform doppler shifts", IEEE J. Oceanic Eng., vol. 33, no. 2, pp. 198 -209, Apr. 2008.

[11] A.E. Abdelkareem, B.S. Sharif, C.C. Tsimenidis, J.A. Neasham, and O.R. Hinton, "Low-complexity doppler compensation for ofdm-based underwater acoustic communication systems", in Proc. MTS/IEEE OCEANS Conf., Santander, Spain, june 2011. 